\title{
Fatty acid profiles of milk from Holstein cows, Jersey cows, buffalos, yaks, humans, goats, camels, and donkeys based on gas chromatography-mass spectrometry
}

\author{
Fengen Wang, ${ }^{1,2,3,4} \odot$ Meiqing Chen, ${ }^{2,4}$ Runbo Luo, ${ }^{2,4}$ Guoxin Huang, ${ }^{2,4} \odot$ Xufang Wu, ${ }^{2,4}$ Nan Zheng, ${ }^{2,4} \odot$ \\ Yangdong Zhang, ${ }^{2,4 *}$ (i) and Jiaqi Wang ${ }^{1,2,4 *}$ (i) \\ ${ }^{1}$ College of Animal Science, Xinjiang Agriculture University, Urumchi 830,091, P. R. China \\ ${ }^{2}$ Key Laboratory of Quality and Safety Control for Milk and Dairy Products of Ministry of Agriculture and Rural Affairs, Institute of Animal Sciences, \\ Chinese Academy of Agricultural Sciences, Beijing 100,193, P. R. China \\ ${ }^{3}$ Institute of Quality Standard and Testing Technology for Agro-Products, Shandong Academy of Agricultural Sciences, Jinan 250,100, P. R. China \\ ${ }^{4}$ Laboratory of Quality and Safety Risk Assessment for Dairy Products of Ministry of Agriculture and Rural Affairs, Institute of Animal Sciences, \\ Chinese Academy of Agricultural Sciences, Beijing 100,193, P. R. China
}

\section{ABSTRACT}

Due to the diversity and limitation of determination methods, published data on the fatty acid (FA) compositions of different milk samples have contributed to inaccurate comparisons. In this study, we developed a high-throughput gas chromatography-mass spectrometry method to determinate milk FA, and the proposed method had satisfactory linearity, sensitivity, accuracy, and precision. We also analyzed the FA compositions of 237 milk samples from Holstein cows, Jersey cows, buffalos, yaks, humans, goats, donkeys, and camels. Holstein, Jersey, goat, and buffalo milks contained high content of even-chain saturated FA, whereas goat milk had higher content of medium- and short-chain FA (MSCFA). Yak and camel milk are potential functional foods due to their high levels of odd- and branchedchain FA and low ratios of n- 6 to n- 3 polyunsaturated FA (PUFA). Human milk contained lower levels of saturated FA, MSCFA, and conjugated linoleic acid, and higher levels of monounsaturated FA and PUFA. As a special nonruminant milk, donkey milk contained low levels of monounsaturated FA and high levels of PUFA and MSCFA. Based on the FA profiles of 8 types of milk, nonruminant milk was distinct from ruminant milk, whereas camel and yak milk were different from other ruminant milks and considered as potential functional foods for balanced human diet.

Key words: milk fatty acid, nonruminant, ruminant, gas chromatography-mass spectrometry

\footnotetext{
Received May 17, 2021.

Accepted September 29, 2021.

*Corresponding authors: zhangyangdong@caas.cn and jiaqiwang@ vip.163.com
}

\section{INTRODUCTION}

Cow milk is an important source of dietary proteins and lipids. Milk fat is rich in bioactive fatty acids (FA), and dairy consumption is associated with a low prevalence of cardiovascular disease and diabetes (Bhavadharini et al., 2020). Fatty acids are generally classified into long-chain fatty acids (LCFA; C $\geq 14$ ) and medium- and short-chain fatty acids (MSCFA; C $\leq 13$ ) according to the number of carbon atoms or SFA, MUFA, and PUFA based on the number of carbon-carbon double bonds. Saturated FA are further classified into branched-chain saturated fatty acids (BCSFA) and straight-chain FA, including even-chain saturated fatty acid (ECSFA) and odd-chain saturated fatty acid (OCSFA). The role of SFA in human health has been comprehensively studied and debated in the last decades, including the effects of LCFA on cardiovascular disease, the beneficial effects of MSCFA on weight control and lipid metabolism, and the positive effects of (OBCFA) on inflammatory conditions (Gómez-Cortés et al., 2018). Unsaturated FA have attracted a lot of attention within the scientific community due to the immunomodulatory properties of CLA; the positive effects of n-3 PUFA on arrhythmia, neurological development, and immune system modulation; and the controversial role of trans MUFA on cardiovascular disease (Dilzer and Park, 2012; Kuhnt et al., 2016; Fuke and Nornberg, 2017). The FA composition of milk fat is also associated with the physical properties and quality of dairy products, and is indicative of the health status of cows (Liu et al., 2018). Therefore, the analysis of FA profiling is necessary and critical in dairy research. However, FA composition of milk fat is quite complex because FA are derived from dietary intake, de novo synthesis in mammary glands, and rumen fermentation. In ruminants, the precursors of $\mathrm{C} 4$ to $\mathrm{C} 16 \mathrm{FA}$ are provided ad- 
ditionally by rumen microbes (Firl et al., 2014; Lanier and Corl, 2015). Some FA, such as CLA and OBCFA, were more often synthesized during rumen microbial fermentation than in animal mammary glands (Fievez et al., 2012; Liu et al., 2018), and thus ruminant milk is the main source of these FA for humans (Gómez-Cortés et al., 2018).

Approximately $400 \mathrm{FA}$ are present in bovine milk (Jensen, 2002), but less than $50 \mathrm{FA}$ have been actually quantified in previous reports (Liu et al., 2018). For FA quantification, researchers have used GC coupled with either a flame ionization detector or MS (Firl et al., 2014). A flame ionization detector, however, has a weak sensitivity. Furthermore, with a flame ionization detector, it is challenging to identify FA in co-eluting peaks. In contrast, MS allows FA identification without the need for standards and has a better sensitivity and accuracy. Fatty acids in milk are present at different levels; GC-based methods often require multiple injections for both major and minor FA to fit the relatively narrow linear ranges (Liu et al., 2018). Thus, a highthroughput and sensitive method is required for FA analysis in milk.

The FA profile of bovine milk has been widely analyzed using GC-based methods (Amores and Virto, 2019; Waktola et al., 2020; Liu et al., 2021), in which FA are converted into FAME. Systematic studies have presented the FA profile in human milk (Cruz-Hernandez et al., 2013; Demmelmair and Koletzko, 2018; Zhao et al., 2018) and compared human and bovine milk (Sokol et al., 2015; He et al., 2016; Wang et al., 2020; Nguyen et al., 2021). The FA compositions of buffalo (Pegolo et al., 2017), yak (Liu et al., 2011), goat (Yurchenko et al., 2018; Toral et al., 2020), camel (Chamekh et al., 2020), and donkey (Martini et al., 2015; Massouras et al., 2017) milk have also been reported. However, the data in the literature are quite fragmented and contribute to inaccurate comparisons due to different determination methods.

In this study, we aimed to develop a high-throughput method for FA determination in milk and to apply it in the analysis of FA profiling from the milk samples of Holstein cows, Jersey cows, buffalos, yaks, humans, goats, camels, and donkeys.

\section{MATERIALS AND METHODS}

\section{Chemicals and Reagents}

Methanol and n-hexane (all HPLC grade) were obtained from Fisher Scientific. We obtained $\mathrm{NaOH}$ (purity $\geq 95 \%$ ) and pyrogallic acid from Macklin. Ammonium hydroxide (purity $\geq 25 \%$ ), ethanol (purity $\geq$ 95\%), isopropanol (purity $\geq 99.5 \%$ ), and acetyl chloride (purity $>99.5 \%$ ) were acquired from Anpel. Ultra-pure water was prepared using a Milli-Q purification system (Millipore).

In the study, the following standards were used: FA solution GLC 617 from Anpel; FAME solution GLC 674 from Nu-Chek Prep; 37-FAME-Mix from Supelco; iso- and anteiso-FAME individual standards from Larodan; C5:0, C7:0, C9:0, and C19:0 FAME individual standards from Dr. Ehrenstorfer; C18:1 cis-6, C18:1 cis-8, C18:1 cis-11, C18:1 trans-6, and C18:1 trans-11 FA individual standards and C18:2 cis-9,trans-11, and C18:2 trans-10,cis-12 FAME individual standards from Anpel; C22:5 cis-4,cis-7,cis-10,cis-13,cis-16, and C22:5 cis-7,cis-10,cis-13,cis-16,cis-19 FAME individual standards from A ChemTek; C12:1 cis-5, C16:1 cis-7, and C19:1 cis-10 FA individual standards from SigmaAldrich; and C10:1 cis-4, C10:1 cis-9, and C10:1 cis-3 FA individual standards from Macklin.

\section{Milk Samples}

In 2020, we collected 237 raw milk samples as follows: 207 milk samples provided by the State Key Laboratory of Animal Nutrition, Institute of Animal Science, Chinese Academy of Agricultural Sciences, and 30 human milk samples donated by volunteer mothers. All samples were frozen immediately after collection and stored at $-20^{\circ} \mathrm{C}$.

Holstein cow milk samples were obtained from Tianjin $(\mathrm{n}=41)$, Inner Mongolia $(\mathrm{n}=7)$, Ningxia autonomous region $(\mathrm{n}=2)$, Shandong province $(\mathrm{n}=3)$, Hebei province $(\mathrm{n}=3)$, and Heilongjiang province $(\mathrm{n}=3)$. Jersey cow milk samples were obtained from Shanghai $(\mathrm{n}=8)$, Heilongjiang province $(\mathrm{n}=5)$, Liaoning province $(\mathrm{n}=9)$, Shandong province $(\mathrm{n}=7)$, Henan province $(\mathrm{n}=2)$, and Guangxi autonomous region $(\mathrm{n}=$ 1). Buffalo milk samples were obtained from Guangxi autonomous region $(\mathrm{n}=9)$. Yak milk samples were obtained from Qinghai province $(\mathrm{n}=18)$ and Sichuan province $(\mathrm{n}=7)$. Goat milk samples were obtained from Shaanxi province $(\mathrm{n}=29)$, Inner Mongolia $(\mathrm{n}=5)$, Henan province $(\mathrm{n}=2)$, and Shandong province $(\mathrm{n}=$ 2 ). Donkey milk samples were obtained from Shandong province $(\mathrm{n}=10)$ and Hebei province $(\mathrm{n}=5)$. Camel milk samples were obtained from Inner Mongolia $(\mathrm{n}=$ $9)$ and Ningxia autonomous region $(\mathrm{n}=20)$. Human milk samples were donated by Chinese mothers from Shandong province $(\mathrm{n}=20)$, Henan province $(\mathrm{n}=6)$, and Hubei province $(\mathrm{n}=4)$.

\section{Sample Preparation}

The frozen milk samples were placed in a water bath at $40^{\circ} \mathrm{C}$ and stirred carefully to avoid frothing. After 
lipid extraction, the FA were converted into FAME according to AOAC International $(2002 ; 996.06)$ and Serafim et al. (2019).

Briefly, a 2-mL milk sample was mixed with $2 \mathrm{~mL}$ of ammonium hydroxide, $1 \mathrm{~mL}$ of ethanol, and $50 \mathrm{mg}$ of pyrogallic acid, and then the mixture was placed in a water bath at $70^{\circ} \mathrm{C}$ for 20 min. Internal standard $\mathrm{C} 10: 1$ cis-4 FA was added after cooling. After the addition of $4 \mathrm{~mL}$ of $\mathrm{n}$-hexane/isopropanol ( $\mathrm{vol} / \mathrm{vol}=3 / 2$ ), the hydrolysate was oscillated and centrifuged $(10,000 \times g$, $4^{\circ} \mathrm{C}$ for $5 \mathrm{~min}$ ) for lipid extraction. We added $2.4 \mathrm{~mL}$ of n-hexane for additional extraction. The extracted n-hexane was collected and mixed with $2 \mathrm{~mL}$ of methanolic $\mathrm{NaOH}(2 \%)$, sealed, and heated at $50^{\circ} \mathrm{C}$ in a water bath for $20 \mathrm{~min}$ for base-methylation, followed by an acid-methylation at $80^{\circ} \mathrm{C}$ in a water bath for $150 \mathrm{~min}$ using $2 \mathrm{~mL}$ of acetyl chlorocarbinol (10\%). After cooling, $5 \mathrm{~mL}$ of n-hexane and $5 \mathrm{~mL}$ of ultra-pure water were added, and n-hexane was subsequently diluted and analyzed by GC-MS.

\section{GC-MS Detection}

Fatty acids were analyzed using an Agilent 7890A GC system equipped with a 7000B MS detector (Agilent Technologies) and a capillary DB-WAX UI column $(30 \mathrm{~m} \times 0.25 \mathrm{~mm} \times 0.25 \mu \mathrm{m})$. The injection volume was $1 \mu \mathrm{L}$, and the split ratio was 50:1. Helium was used as the carrier gas at a constant pressure of $7 \mathrm{psi}$, and the total run time was $94 \mathrm{~min}$. The inlet temperature was $250^{\circ} \mathrm{C}$, and the oven temperature had the following program: $60^{\circ} \mathrm{C}$ held for $2 \mathrm{~min}$, increased by $30^{\circ} \mathrm{C} / \mathrm{min}$ to $120^{\circ} \mathrm{C}$, increased by $4^{\circ} \mathrm{C} / \mathrm{min}$ to $160^{\circ} \mathrm{C}$, held at $160^{\circ} \mathrm{C}$ for $40 \mathrm{~min}$, increased by $4^{\circ} \mathrm{C} / \mathrm{min}$ to $200^{\circ} \mathrm{C}$, held at $200^{\circ} \mathrm{C}$ for $20 \mathrm{~min}$, increased by $20^{\circ} \mathrm{C} / \mathrm{min}$ to $240^{\circ} \mathrm{C}$, and held at $240^{\circ} \mathrm{C}$ for $8 \mathrm{~min}$.

The temperature of the transfer line was set to $250^{\circ} \mathrm{C}$, and the solvent delay was $2 \mathrm{~min}$. The temperature of the MS ion source was set to $230^{\circ} \mathrm{C}$, and the ionizing energy was $70 \mathrm{eV}$. Qualitative and quantitative analyses of FAME were performed in the selected ion monitoring mode (Table 1). Peak identification of FAME was based on retention time and the characteristic ions, including 1 quantitative ion and 3 qualitative ions, and structure verification was applied in full scan mode and compared with the spectra from NIST database (National Institute of Standards and Technology, https://doi .org/10.18434/T4H594), a commercially available MS database (Albergamo et al., 2016). Based on the peak area of the quantitative ion, FAME quantification was performed using standard curves of each external standard and calibrated using the internal standard. As the differences in the response factors of FAME should be considered in GC-MS selected ion monitoring method
(Thurnhofer and Vetter, 2005; Hauff and Vetter, 2009), standard curves and linear ranges of each FAME were established. To improve sensitivity, the quantitative ion of each FAME was selected at the best signal-to-noise ratio, and the runtime was divided into 15 time windows to scan the selected characteristic ions, in which the dwell time of each ion was $>9 \mathrm{~ms}$, and the scanning frequency was $>8$ cycle/s. The absolute concentrations of FAME determined by GC-MS were normalized as relative amounts (FA mass percentages, \%) to reduce the error caused by sample nonuniformity and compared with the data reported in the literature.

\section{Method Validation}

The proposed method was validated for sensitivity, linearity, accuracy, and precision according to the validation guide (ICH, 2005). The limit of quantitation (LOQ) of each FAME was calculated based on signalto-noise ratio of 10 . The standard curve and linear range for each FAME were established, and at least 5 different concentrations were used to determine the regression equation within a range of 0.02 to $150 \mathrm{mg} / \mathrm{L}$. We prepared a whey solution by mixing $1 \mathrm{~g}$ of whey powder with $30 \mathrm{~mL}$ of ultra-pure water. We conducted recovery experiments, in which $2 \mathrm{~mL}$ of whey solution was spiked with 3 different concentrations of FA solution GLC 617.

\section{Statistical Analysis}

Peak areas were obtained using Qualitative Analysis B.04.00 (MassHunter Workstation Software, Agilent Technologies). The data were analyzed and sorted using Excel 2019. Principal component analysis (PCA) was performed using GraphPad Prism 9 (GraphPad Software). Heatmap clusters were analyzed using the $\mathrm{R}$ package version 1.0.12 (https://www.r-project.org/).

\section{RESULTS AND DISCUSSION}

\section{Method Validation}

We detected 69 FAME simultaneously with acceptable resolution (Figure 1a and b). However, we had considerable difficulty separating individual C18:1 FAME with numerous positional and geometric isomers, as previously reported (Amores and Virto, 2019). The C18:1 FAME isomers were not well separated in the chromatogram (Figure 1c) and were labeled as $\Sigma \mathrm{C} 18: 1$ for the sake of accuracy and precision. In addition, limited by the available nonconjugated C18:2 FAME standards, only $\mathrm{C} 18: 2$ cis-9,cis- 12 , and $\mathrm{C} 18: 2$ trans9,trans-12 were quantified and labeled (Figure 1d), 
Wang et al.: FATTY ACID PROFILES OF 8 TYPES OF MILK

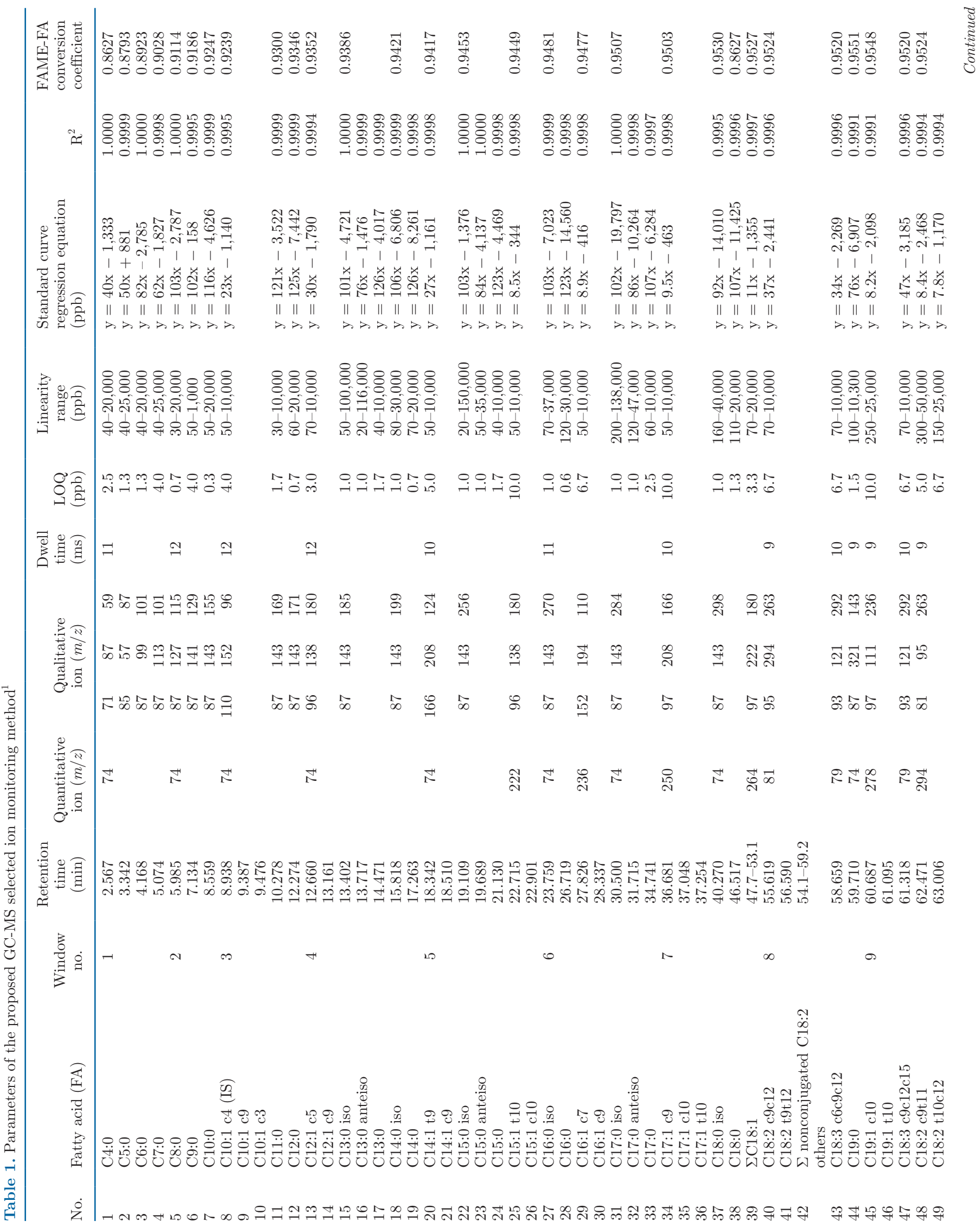




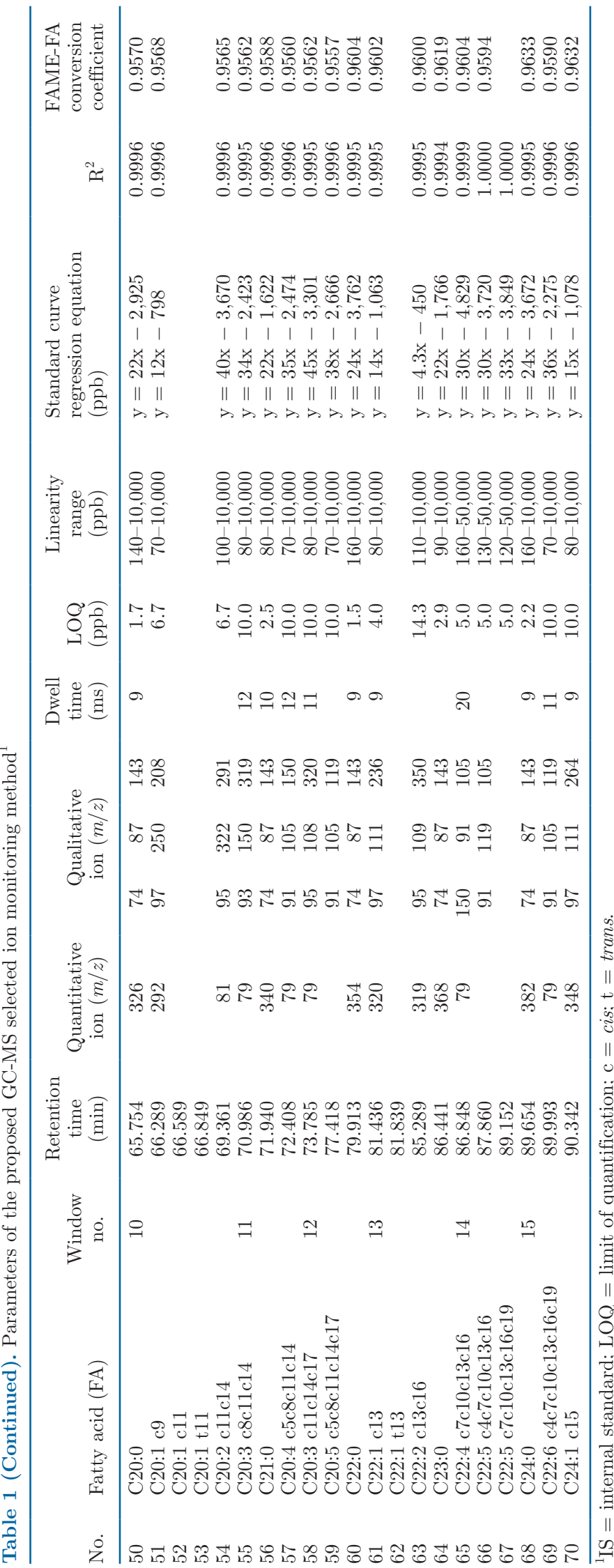

whereas the other C18:2 FAME isomers were quantified together and labeled as $\Sigma$ nonconjugated C18:2 others for the sake of simplicity.

As shown in Table 1, the LOQ of FAME ranged from 0.3 to $14.3 \mu \mathrm{g} / \mathrm{L}$, corresponding to 8 to $358 \mu \mathrm{g} / \mathrm{L}$ of FA from milk samples. The minimum relative amounts ranged from 0.00002 to $0.0009 \%$, assuming a total milk fat content of $\sim 4 \%$. The regression equations of all FAME standard curves were established at a wide range $(0.02-150 \mu \mathrm{g} / \mathrm{mL})$, with determination coefficients $>0.9991$. As most of FAME isomers had similar abundance in characteristic ions, the same regression equations were used for their calculation. The recovery rates ranged from 76.6 to $109.3 \%$ with variable coefficient ranging from 0.5 to $11.7 \%$ (Supplemental Table S1; https://data.mendeley.com/datasets/j9h7967pcy/ 1, Wang et al., 2021). Therefore, our high-throughput method had adequate linearity, sensitivity, accuracy, and precision.

\section{Fatty Acid Profile of Milk}

The proposed method was used to determine the FA composition of raw milk samples from Holstein cows, Jersey cows, buffalos, yaks, humans, goats, donkeys, and camels. Fatty acids were classified as ECSFA, OCSFA, BCSFA, MUFA, and PUFA, and more than $60 \mathrm{FA}$ were detected in each milk sample (Table 2).

According to the pie charts (Figure 2), the milk samples from Holstein cows, Jersey cows, goats, and buffalos had comparable ECSFA (57.3-58.8\%), OCSFA (1.3-1.8\%), BCSFA (1.4-1.9\%), MUFA (33.1-35.3\%), and PUFA (3.8-5.1\%). Previous studies have reported higher SFA levels (61.3-73.9\%), lower MUFA levels (22.3-33.0\%), and comparable BCSFA (1.1-2.7\%) and PUFA (2.1-5.5\%) levels (Dreiucker and Vetter, 2011; Kairenius et al., 2015; Pegolo et al., 2017; Teng et al., 2017; Yurchenko et al., 2018). Compared with such profiles, the levels of OCSFA, BCSFA, MUFA, and PUFA in yak and camel milk increased and the levels of ECSFA decreased in our study. In ruminant milk samples, the highest levels of OCSFA (2.8\%), BCSFA (3.0\%), and PUFA (6.3\%) were detected in yak milk, whereas the highest levels of MUFA (40.7\%) and lowest levels of ECSFA (48.6\%) were present in camel milk. Previous studies have reported that higher levels of MUFA (25.7-32.8\%), PUFA (3.9-8.1\%), and BCSFA (2.2-3.0\%) were found in camel milk instead of other ruminant milk, as well as lower levels of SFA (54.5-64.1\%; Dreiucker and Vetter, 2011; Teng et al., 2017; Chamekh et al., 2020). Fatty acid profiles were different between human and donkey milk and ruminant milk. As shown in Table 2, human milk had the lowest levels of ECSFA (31.3\%), OCSFA (0.4\%), and BCSFA 

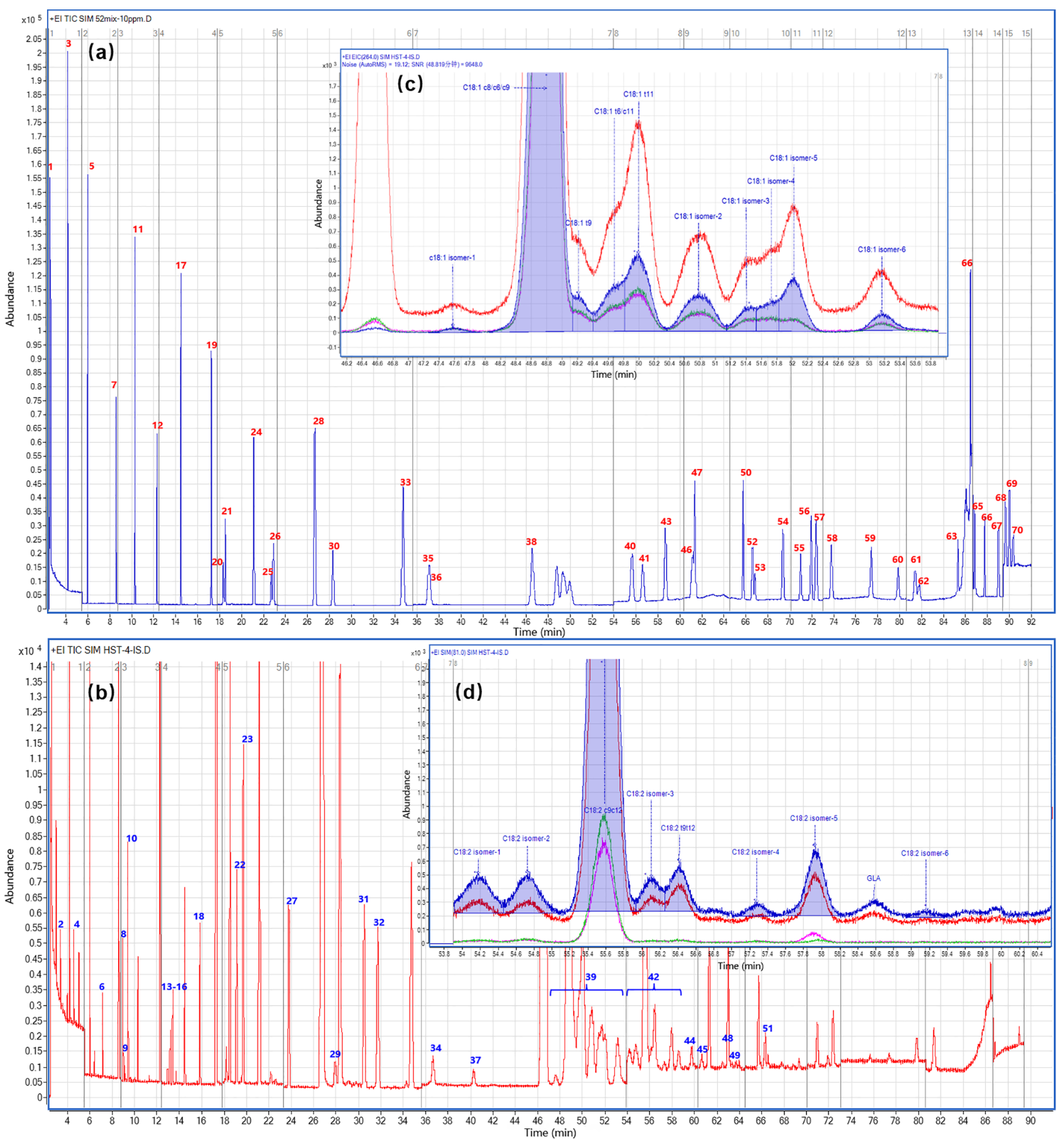

Figure 1. (a) Total ion chromatograms (TIC) of the FAME from mixed standards solution. (b) TIC of the FAME from Holstein cow milk. (c) Elected ion chromatograms (EIC) of C18:1 FAME from Holstein cow milk. (d) EIC of C18:2 FAME from Holstein cow milk. The serial numbers of fatty acids in Table 1 are labeled on the chromatograms. 


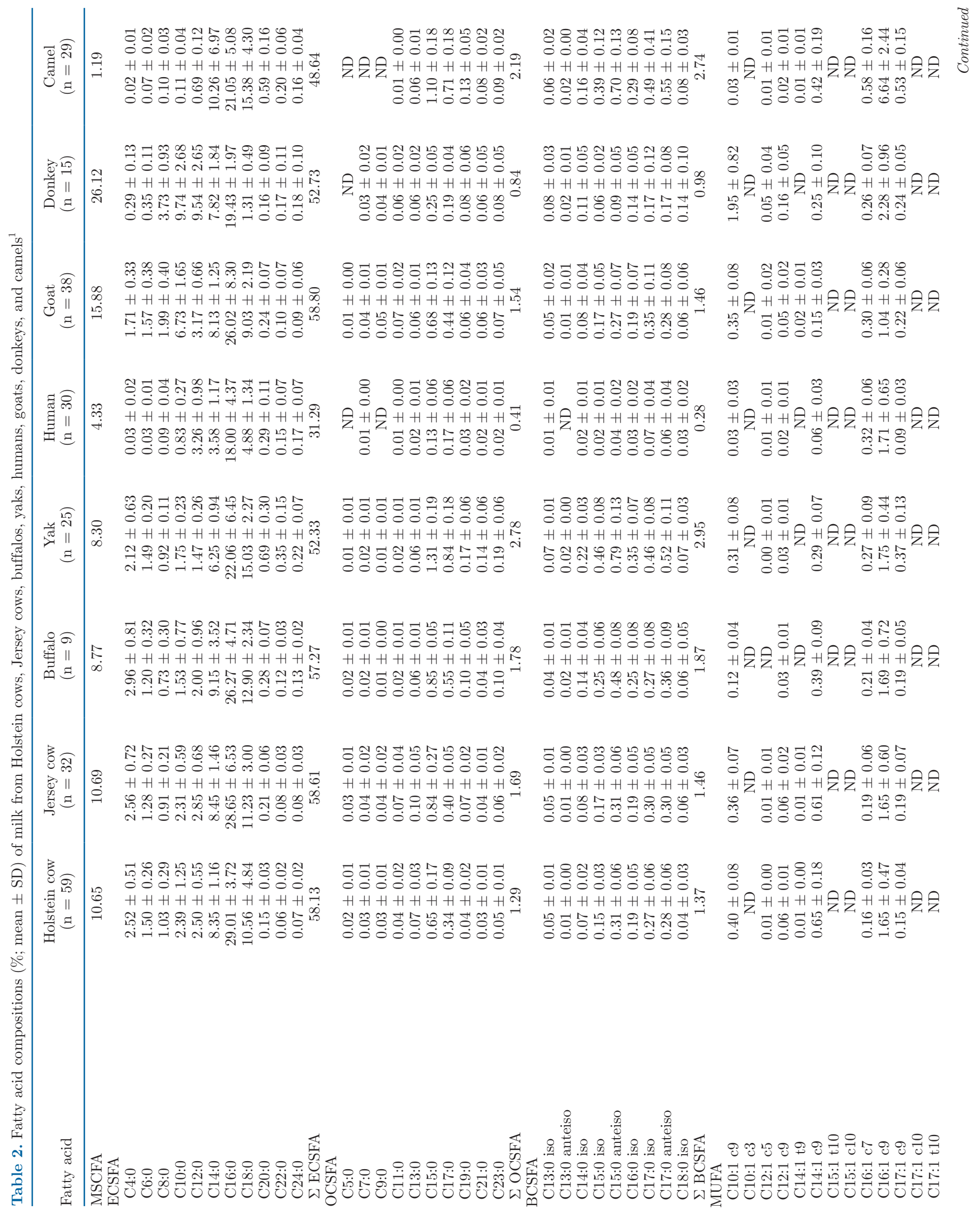




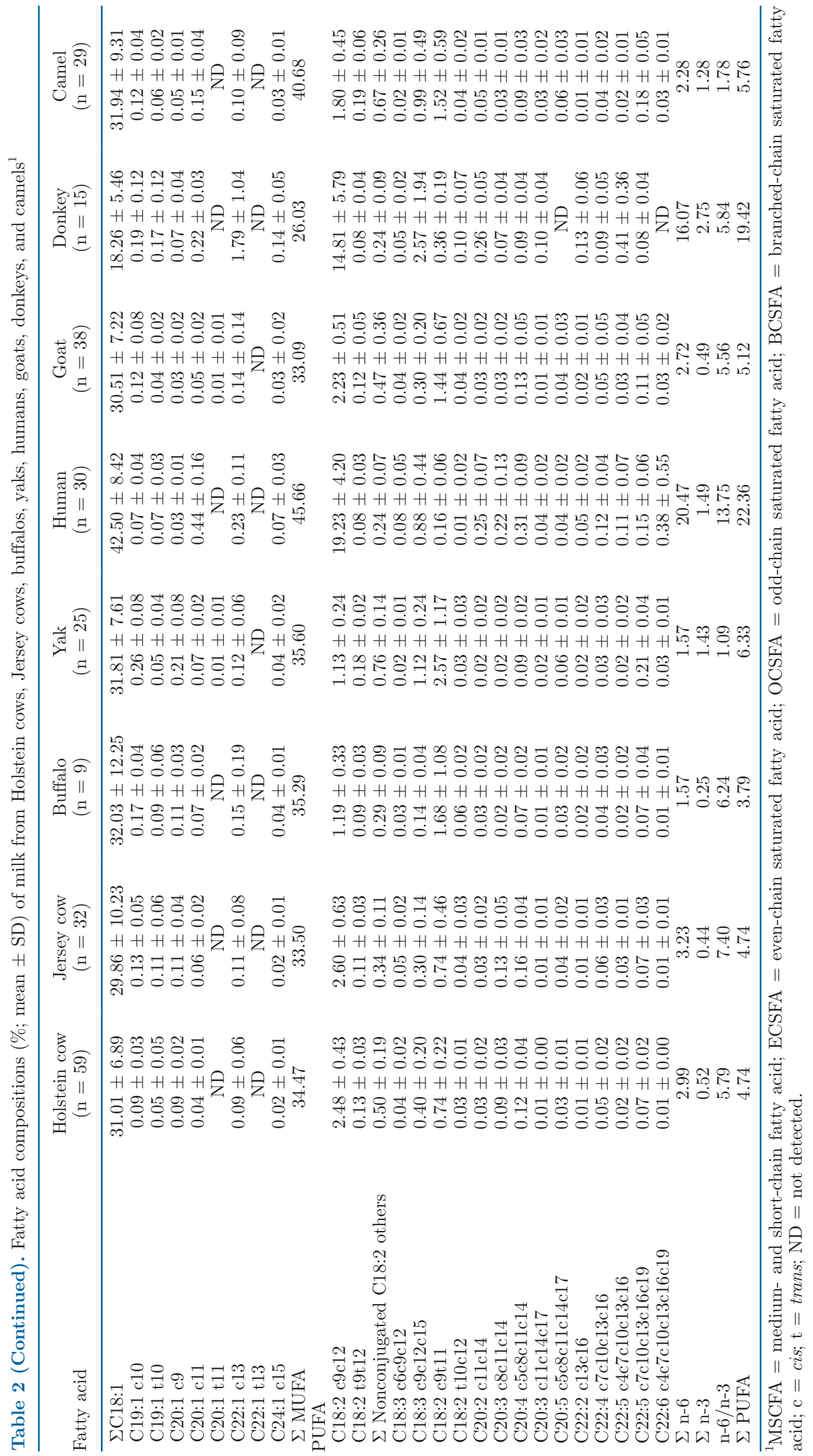


(0.3\%), and the highest levels of MUFA (45.7\%) and PUFA (22.4\%). Fatty acid levels in human milk were quite variable (Dreiucker and Vetter, 2011; Teng et al., 2017; Wang et al., 2020; Nguyen et al., 2021). For example, SFA levels range from 29.0 to $56.8 \%$, MUFA levels range from 20.6 to $41.3 \%$, and PUFA levels range from 19.6 to $50.5 \%$, mainly due to differences in the diet. In donkey milk, the levels of PUFA reached 19.4\%, which exceeded the values reported in the literature (12.30-16.11\%), whereas the levels of MUFA (26.0\%) were below those previously reported (32.67-41.68\%; Martini et al., 2015).

As shown in Table 2, Holstein, Jersey, buffalo, and yak milk contained comparable MSCFA, which were $10.6 \%, 10.7 \%, 8.8 \%$, and $8.3 \%$, respectively. Compared with bovine milk, donkey milk $(26.1 \%)$ and goat milk (15.9\%) had higher MSCFA levels, and camel milk $(1.2 \%)$ and human milk (4.3\%) had lower MSCFA levels, consistent with past findings (Zou et al., 2013; Chamekh et al., 2020).

\section{Comparison Among SFA}

According to the PCA diagram (Figure 3b), SFA composition in human, camel, and yak milk were different from that of other milk samples. As shown in Table 2, the levels of individual SFA were lower in human milk than in other milk samples, except for C12:0, C20:0, C22:0, and C24:0. Moreover, the levels of C15:0 and C18:0 were much higher in yak milk $(1.31 \%$ and $15.03 \%$, respectively) and camel milk (1.10\% and $15.38 \%$, respectively) than in other samples. The highest levels of C17 to C24 SFA were detected in yak milk, whereas the highest levels of C14:0 (10.26\%) and the lowest levels of $\mathrm{C} 4$ to $\mathrm{C} 12 \mathrm{SFA}$ were detected in camel milk. Donkey milk had comparatively higher levels of C8 to C13 SFA, especially C8:0 (3.73\%), C10:0 (9.74\%), and $\mathrm{C} 12: 0$ (9.54\%), and lower levels of C15 to C18 SFA, particularly C18:0 (1.31\%), and extremely low levels of C4 to C6 SFA. Higher levels of C6 to C11 SFA, especially C6:0 (1.57\%), were found in goat milk. Holstein, Jersey, and buffalo milk had similar levels of ECSFA, with higher levels of C4:0 and C16:0, whereas Holstein cow had very low levels of C19 to C24 SFA.

Odd- and branched-chain FA have received considerable attention due to their nutritional benefits and functional properties (Vlaeminck et al., 2015; GómezCortés et al., 2018). As shown in Supplemental Figure S1 (https://data.mendeley.com/datasets/j9h7967pcy/ 1, Wang et al., 2021), the highest levels of OBCFA were found in yak and camel milk, whereas the lowest levels were detected in human milk, consistent with the findings of Teng et al. (2017). There was an abundance of
C15 to C23 OCSFA and C14 to C17 BCSFA in yak and camel milk. Furthermore, C5 to C13 OCSFA in Jersey cow milk; C9:0 and C11:0 in goat milk; and C13:0 iso, C13:0 anteiso, and C18:0 iso in donkey milk were the most abundant.

\section{Comparison Among MUFA}

According to PCA (Figure 3c), MUFA compositions in human and donkey milk were different from ruminant milk. Table 2 shows that the highest levels of MUFA were present in human milk, whereas the lowest levels were present in donkey milk. The total level of $\mathrm{C} 18: 1$, which represented the most abundant MUFA in the milk samples, was the highest in human milk $(42.50 \%)$ and the lowest in donkey milk (18.26\%), whereas researchers have reported that MUFA levels are 18.2 to $38.8 \%$ in human milk (Dreiucker and Vetter, 2011; Teng et al., 2017; Wang et al., 2020; Nguyen et al., 2021) and 21.9 to $27.9 \%$ in donkey milk (Martini et al., 2015). The total levels of C18:1 in ruminant milk ranged from $29.86 \%$ (Jersey milk) to $32.03 \%$ (buffalo milk). In addition to C18:1, C16:1 cis-9 was another major MUFA in the milk samples. C16:1 cis-9 is an n-7 FA, which has drawn considerable attention due to its functional properties (Frigolet and Gutiérrez-Aguilar, 2017). The highest levels of C16:1 cis-9 were detected in camel milk $(6.64 \%)$, consistent with the findings of Chamekh et al. (2020) and Teng et al. (2017). In goat and donkey milk, C16:1 cis-9 levels were 1.04 and $2.28 \%$, respectively.

The diversity of MUFA in human milk was limited, and the main MUFA were C16:1, C18:1, and C20:1, as previously reported (Dreiucker and Vetter, 2011; Teng et al., 2017; Wang et al., 2020; Nguyen et al., 2021). The highest levels of C10:1, C12:1, C22:1, and C24:1 were detected in donkey milk, especially C10:1 cis-9 $(1.95 \%)$ and $\mathrm{C} 22: 1$ cis-13 (1.79\%). The C10:1 cis-9 in milk samples was never mentioned in previous studies, whereas the levels of $\mathrm{C} 22: 1$ cis-13 were lower in literature (Martini et al., 2015) than our results. In addition, C14:1 in Holstein and Jersey cow milk, C16:1 and C17:1 in camel milk, and C19:1 and C20:1 in yak milk were the most abundant.

\section{Comparison Among PUFA}

According to the PCA diagram (Figure 3d), PUFA composition in human and donkey milk were different from ruminant milk. As shown in Table 2, higher levels of PUFA were detected in human milk $(22.36 \%)$ and donkey milk (19.42\%), whereas PUFA levels in ruminant milk ranged from $3.79 \%$ (buffalo milk) to $6.33 \%$ 


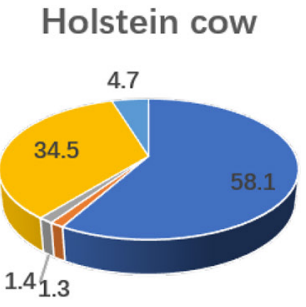

Human

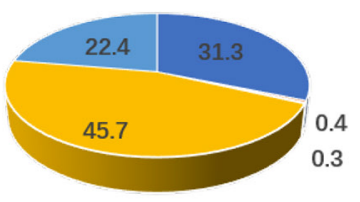

\section{- ECSFA " OCSFA | BCSFA " MUFA $\mid$ PUFA}

Jersey cow



Goat

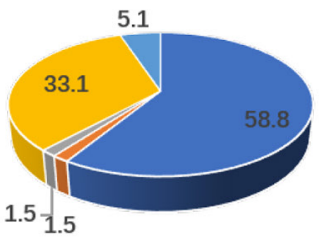

Buffalo

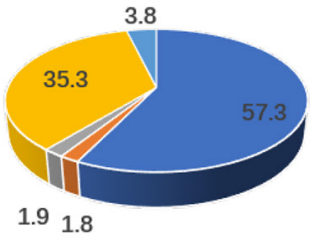

Donkey

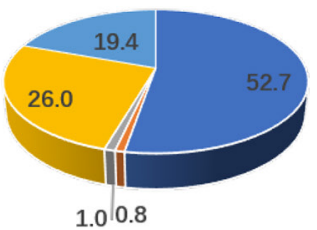

Yak

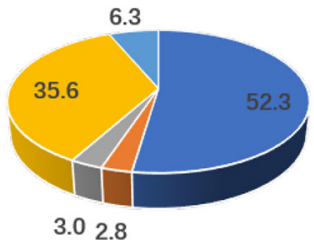

Camel

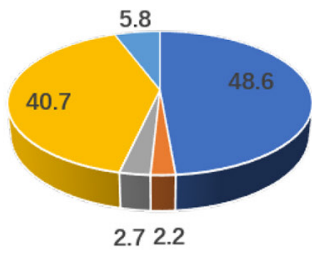

Figure 2. Pie charts of the fatty acids in milk samples from Holstein cows, Jersey cows, buffalos, yaks, humans, goats, donkeys, and camels. ECSFA = even-chain saturated fatty acid; OCSFA = odd-chain saturated fatty acid; BCSFA = branched-chain saturated fatty acid.

(a)

PC scores

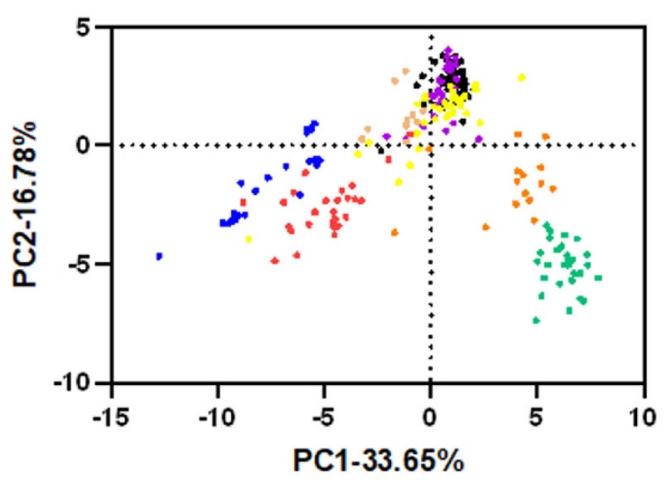

(c)

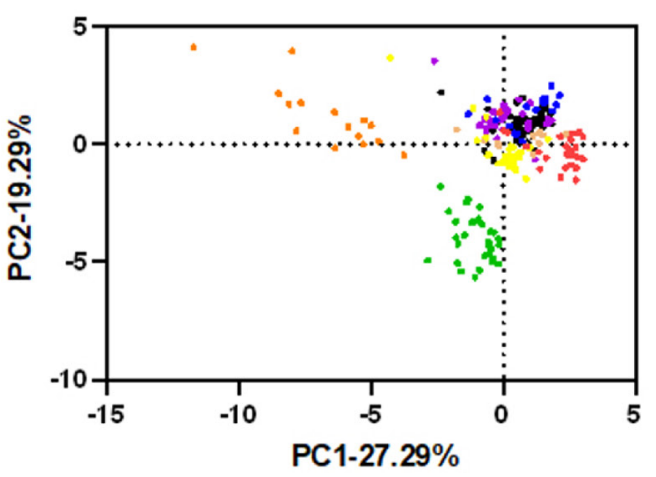

(b) PC scores
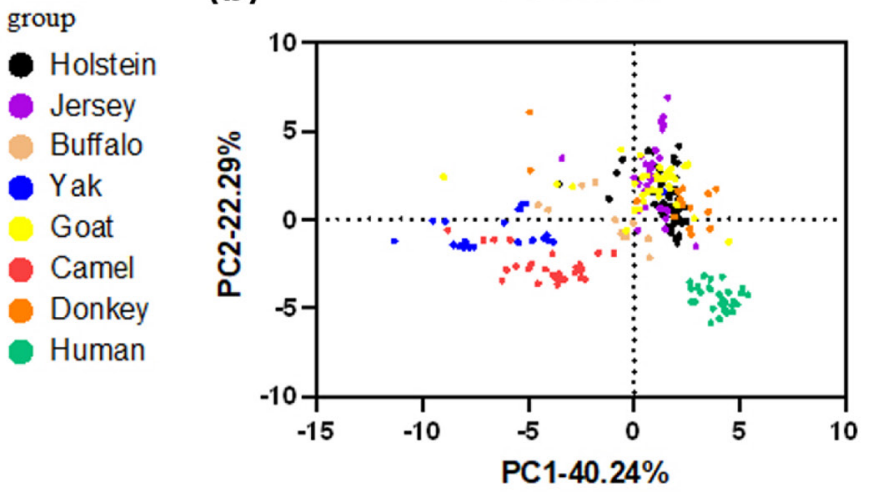

group

- Holstein

- Jersey

- Buffalo

- Yak

Goat

Camel

- Donkey

- Human (d)

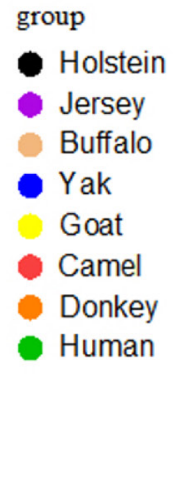

PC scores

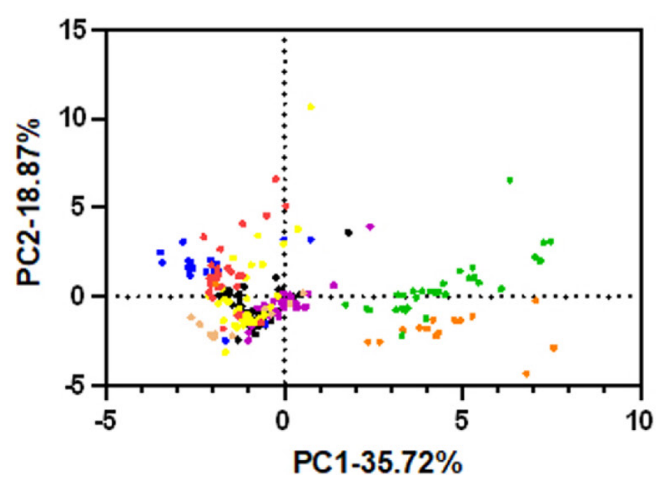

group

- Holstein

- Jersey

Buffalo

- Yak

Goat

- Camel

Donkey

- Human

Figure 3. Principal component (PC) analysis diagrams of the fatty acid composition in milk samples from Holstein cows, Jersey cows, buffalos, yaks, humans, goats, donkeys, and camels. (a) All fatty acids, (b) SFA, (c) MUFA, and (d) PUFA. 
(yak milk). In ruminal animals, PUFA from the diet are simply isomerized as the first step of ruminal biohydrogenation and thus effected the milk FA composition (Dreiucker and Vetter, 2011). The predominant PUFA in human milk (19.23\%) and donkey milk (14.81\%) was C18:2 cis-9,cis-12, whereas C18:2 cis-9,cis-12 levels in ruminant milk ranged from $1.13 \%$ (yak milk) to $2.60 \%$ (Jersey cow milk). Additionally, C18:2 cis-9,trans-11, which has multiple bioactive effects (Fuke and Nornberg, 2017; Shokryazdan et al., 2017), was the predominant CLA isomer in the milk samples. Higher levels of C18:2 cis-9,trans-11 were detected in ruminant milk (from $0.74 \%$ in both Holstein and Jersey cow milk to $2.57 \%$ in yak milk), whereas lower levels were present in human milk $(0.16 \%)$ and donkey milk $(0.36 \%)$. The highest levels of C18:2 trans-10,cis-12 were detected in donkey milk $(0.10 \%)$. Conjugated linoleic acid is mainly derived from the isomerization of dietary C18:2 cis-9,cis-12 in rumen or the desaturation of C18:1 trans-11 in vivo (Siurana et al.,2018). The CLA in donkey milk is unique and noteworthy, especially C18:2 trans-10, cis-12.

The n-3 PUFA, including C18:3 cis-9,cis-12,cis-15, C20:5 cis-5,cis-8,cis-11,cis-14,cis-17, C22:5 cis-7,cis10,cis-13,cis-16,cis-19, and C22:6 cis-4,cis-7,cis-10,cis13,cis-16,cis-19, have beneficial effects on the neurological and cardiovascular systems (Breslow, 2006; Ruiz-Rodriguez et al., 2010; Mozaffarian and Wu,

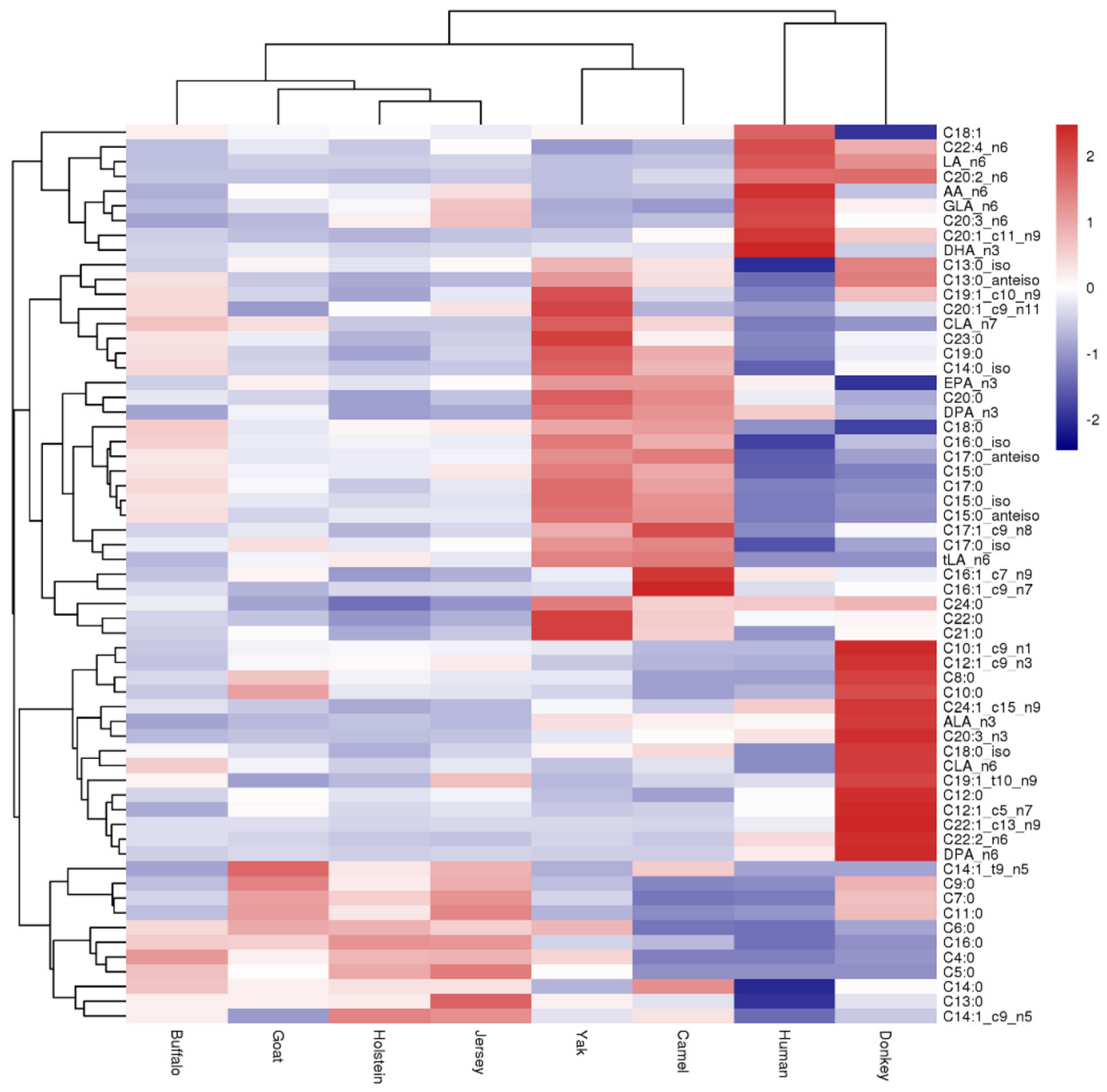

Figure 4. Heatmap of the fatty acids in milk samples from Holstein cow, Jersey cow, buffalo, yak, human, goat, donkey, and camel. c $=$ cis; $\mathrm{t}=$ trans. $\mathrm{EPA}=\mathrm{C} 20: 5 \mathrm{c} 5 \mathrm{c} 8 \mathrm{c} 11 \mathrm{c} 14 \mathrm{c} 17 ; \mathrm{DPA}=\mathrm{C} 22: 5 \mathrm{c} 7 \mathrm{c} 10 \mathrm{c} 13 \mathrm{c} 16 \mathrm{c} 19$ (n-3) and $\mathrm{C} 22: 5 \mathrm{c} 4 \mathrm{c} 7 \mathrm{c} 10 \mathrm{c} 13 \mathrm{c} 16$ (n-6); GLA = C18:3 c6c9c12; ALA = $\mathrm{C} 18: 3 \mathrm{c} 9 \mathrm{c} 12 \mathrm{c} 15$. 
2012). The n-3 PUFA must be derived from the diet because they cannot be synthesized by the human body (Brown and Skeaff, 2017). The lowest and highest levels of C18:3 cis-9,cis-12,cis-15, the prominent n-3 PUFA in the milk samples, were detected in buffalo milk $(0.14 \%)$, which was consistent with the literature (Teng et al., 2017), and in donkey milk (2.57\%). Moreover, the highest levels of C20:3 cis-11,cis-14,cis-17 were detected in donkey milk $(0.10 \%)$; however, C20:5 cis-5,cis-8,cis-11,cis-14,cis-17 and $\mathrm{C} 22: 6$ cis-4,cis-7,cis10, cis-13, cis-16, cis-19 were not detected. Martini et al. (2015) reported that donkey milk contains $0.01 \%$ C20:5 cis-5, cis-8,cis-11,cis-14,cis-17 and $0.03 \%$ C22:6 cis4,cis-7,cis-10,cis-13,cis-16,cis-19. In addition, the highest levels of C22:6 cis-4,cis-7,cis-10,cis-13,cis-16,cis-19 were present in human milk $(0.38 \%)$, and the highest levels of C20:5 cis-5,cis-8,cis-11,cis-14,cis-17 and C22:5 cis-7,cis-10,cis-13,cis-16,cis-19 were found in yak milk $(0.06 \%$ and $0.21 \%$, respectively) and camel milk $(0.06 \%$ and $0.18 \%$, respectively). Excessive intake of n-6 PUFA increases the risk of several chronic diseases (Wu et al., 2010). A range of n-6 to n- 3 ratios is recommended to maintain an adequate FA balance. The highest levels of $\mathrm{C} 18: 3$ cis-6,cis-9,cis-12, C20:2 cis-11,cis-14, C20:3 cis-8,cis-11,cis-14, C20:4 cis-5,cis-8,cis-11,cis-14, and C22:4 cis-7,cis-10,cis-13,cis-16 were found in human milk, and the highest levels of $\mathrm{C} 22: 2$ cis-13, cis-16 and C22:5 cis-4,cis-7,cis-10,cis-13,cis-16 were present in donkey milk. The n- 6 to n- 3 ratio was 13.75 in human milk, 1.09 in yak milk, and 1.78 in camel milk. Therefore, yak and camel milk are potential functional foods (Teng et al., 2017).

\section{Cluster Analysis}

We analyzed the milk samples based on the heatmap and PCA results. Figure 3a shows that human and donkey milk were obviously different from ruminant milk, whereas camel and yak milk were far away from the others (Holstein, Jersey, buffalo, and goat milk), which was consistent with the cluster relationship in the heatmap (Figure 4). Combined with the heatmap and the database (Table 2), the FA fingerprints of the 8 types of milk were established, which presented the distinction in abundance of individual FA and could be used in authenticity identification of milk species (Rebechi et al., 2016).

\section{CONCLUSIONS}

We developed a GC-MS method to simultaneously analyze $69 \mathrm{FA}$ in milk samples, with good linearity, sensitivity, accuracy, and precision. Using this high- throughput method, we comprehensively and accurately analyzed the FA compositions of eight types of milk. Holstein cow, Jersey cow, goat, and buffalo milks were similar in FA profiles and rich in ECSFA, whereas goat milk had higher content of MSCFA than the other 3. Yak and camel milks were rich in OBCFA and n-3 PUFA, and considered as potential functional foods for balanced human diet, whereas camel milk also contained a high content of C16:1 c9 and low levels of MSCFA. Different from ruminant milk, human milk had low levels of SFA, MSCFA, and CLA, and high levels of MUFA and PUFA. Donkey milk, as a special nonruminant milk, contained low levels of MUFA and high levels of PUFA and MSCFA.

\section{ACKNOWLEDGMENTS}

This study was supported by the Scientific Research Project for Major Achievements of the Agricultural Science and Technology Innovation Program (No. CAASZDXT2019004, Beijing, China), the Agricultural Science and Technology Innovation Program (ASTIP-IAS12, Beijing, China), and State Key Laboratory of Animal Nutrition (2004DA125184G2108, Beijing, China). The authors have not stated any conflicts of interest.

\section{REFERENCES}

Albergamo, A., F. Rigano, G. Purcaro, A. Mauceri, S. Fasulo, and L. Mondello. 2016. Free fatty acid profiling of marine sentinels by nanoLC-EI-MS for the assessment of environmental pollution effects. Sci. Total Environ. 571:955-962. https://doi.org/10.1016/ j.scitotenv.2016.07.082.

Amores, G., and M. Virto. 2019. Total and free fatty acids analysis in milk and dairy fat. Separations 6:14. https://doi.org/10.3390/ separations6010014.

AOAC International. 2002. AOAC Official method 996.06. Fat (Total, Saturated, and Unsaturated) in Foods. Association of Official Analytical Chemists.

Bhavadharini, B., M. Dehghan, A. Mente, S. Rangarajan, P. Sheridan, V. Mohan, R. Iqbal, R. Gupta, S. Lear, E. Wentzel-Viljoen, A. Avezum, P. Lopez-Jaramillo, P. Mony, R. P. Varma, R. Kumar, J. Chifamba, K. F. Alhabib, N. Mohammadifard, A. Oguz, F. Lanas, D. Rozanska, K. B. Bostrom, K. Yusoff, L. P. Tsolkile, A. Dans, A. Yusufali, A. Orlandini, P. Poirier, R. Khatib, B. Hu, L. Wei, L. Yin, A. Deeraili, K. Yeates, R. Yusuf, N. Ismail, D. Mozaffarian, K. Teo, S. S. Anand, and S. Yusuf. 2020. Association of dairy consumption with metabolic syndrome, hypertension and diabetes in 147812 individuals from 21 countries. BMJ Open Diabetes Res. Care 8:e000826. https://doi.org/10.1136/bmjdrc-2019-000826.

Breslow, J. L. 2006. n-3 fatty acids and cardiovascular disease. Am. J. Clin. Nutr. 83(Suppl):1477S-1482S. https://doi.org/10.1093/ ajcn/83.6.1477S.

Brown, R., and S. Skeaff. 2017. Nutrition Society of New Zealand Annual Conference Held in Christchurch, New Zealand, 8-9 December 2016. Nutrients 9:348. https://doi.org/10.3390/nu9040348.

Chamekh, L., M. Calvo, T. Khorchani, P. Castro-Gómez, M. Hammadi, J. Fontecha, and M. H. Yahyaoui. 2020. Impact of management system and lactation stage on fatty acid composition of camel milk. J. Food Compos. Anal. 87:103418. https://doi.org/10 .1016/j.jfca.2020.103418. 
Cruz-Hernandez, C., S. Goeuriot, F. Giuffrida, S. K. Thakkar, and F. Destaillats. 2013. Direct quantification of fatty acids in human milk by gas chromatography. J. Chromatogr. A 1284:174-179. https://doi.org/10.1016/j.chroma.2013.01.094.

Demmelmair, H., and B. Koletzko. 2018. Lipids in human milk. Best Pract. Res. Clin. Endocrinol. Metab. 32:57-68. https://doi.org/10 $.1016 /$ j.beem.2017.11.002.

Dilzer, A., and Y. Park. 2012. Implication of conjugated linoleic acid (CLA) in human health. Crit. Rev. Food Sci. Nutr. 52:488-513. https://doi.org/10.1080/10408398.2010.501409.

Dreiucker, J., and W. Vetter. 2011. Fatty acids patterns in camel, moose, cow and human milk as determined with GC/MS after silver ion solid phase extraction. Food Chem. 126:762-771. https:/ /doi.org/10.1016/j.foodchem.2010.11.061.

Fievez, V., E. Colman, J. M. Castro-Montoya, I. Stefanov, and B. Vlaeminck. 2012. Milk odd- and branched-chain fatty acids as biomarkers of rumen function-An update. Anim. Feed Sci. Technol. 172:51-65. https://doi.org/10.1016/j.anifeedsci.2011.12.008.

Firl, N., H. Kienberger, and M. Rychlik. 2014. Validation of the sensitive and accurate quantitation of the fatty acid distribution in bovine milk. Int. Dairy J. 35:139-144. https://doi.org/10.1016/j .idairyj.2013.11.007.

Frigolet, M. E., and R. Gutiérrez-Aguilar. 2017. The role of the novel lipokine palmitoleic acid in health and disease. Adv. Nutr. 8:173s$181 \mathrm{~s}$.

Fuke, G., and J. L. Nornberg. 2017. Systematic evaluation on the effectiveness of conjugated linoleic acid in human health. Crit. Rev. Food Sci. Nutr. 57:1-7. https://doi.org/10.1080/10408398.2012 .716800 .

Gómez-Cortés, P., M. Juárez, and M. A. de la Fuente. 2018. Milk fatty acids and potential health benefits: An updated vision. Trends Food Sci. Technol. 81:1-9. https://doi.org/10.1016/j.tifs.2018.08 .014 .

Hauff, S., and W. Vetter. 2009. Quantitation of cis- and trans-monounsaturated fatty acids in dairy products and cod liver oil by mass spectrometry in the selected ion monitoring mode. J. Agric. Food Chem. 57:3423-3430. https://doi.org/10.1021/jf803665u.

He, Y.-B., H.-W. Ren, Y.-T. Cao, H.-J. Li, Z. Zhang, and N. Liu. 2016. Comparing the composition and trend of fatty acid in human milk with bovine milk and infant formula in northeast region of China. CYTA J. Food 14:632-638. https://doi.org/10.1080/19476337 .2016.1188858

ICH. 2005. ICH Harmonized Tripartite Guideline. Validation of Analytical Procedures: Text and Methodology Q2(R1). ICH.

Jensen, R. G. 2002. The composition of bovine milk lipids: January 1995 to December 2000. J. Dairy Sci. 85:295-350. https://doi.org/ 10.3168/jds.S0022-0302(02)74079-4.

Kairenius, P., A. Ärölä, H. Leskinen, V. Toivonen, S. Ahvenjärvi, A. Vanhatalo, P. Huhtanen, T. Hurme, J. M. Griinari, and K. J. Shingfield. 2015. Dietary fish oil supplements depress milk fat yield and alter milk fatty acid composition in lactating cows fed grass silage-based diets. J. Dairy Sci. 98:5653-5671. https://doi.org/10 .3168/jds.2015-9548.

Kuhnt, K., C. Degen, and G. Jahreis. 2016. Evaluation fo the impact of ruminant trans fatty acids on human health: Important aspects to consider. Crit. Rev. Food Sci. Nutr. 56:1964-1980. https://doi .org/10.1080/10408398.2013.808605.

Lanier, J. S., and B. A. Corl. 2015. Challenges in enriching milk fat with polyunsaturated fatty acids. J. Anim. Sci. Biotechnol. 6:26. https://doi.org/10.1186/s40104-015-0025-0.

Liu, H. N., F. Z. Ren, L. Jiang, Z. L. Ma, H. J. Qiao, S. S. Zeng, B. Z. Gan, and H. Y. Guo. 2011. Short communication: Fatty acid profile of yak milk from the Qinghai-Tibetan Plateau in different seasons and for different parities. J. Dairy Sci. 94:1724-1731. https://doi.org/10.3168/jds.2010-3749.

Liu, X.-M., Y. Zhang, Y. Zhou, G.-H. Li, B.-Q. Zeng, J.-W. Zhang, and X.-S. Feng. 2021. Progress in Pretreatment and Analysis of Fatty Acids in Foods: An Update since 2012. Separ. Purif. Rev. 50:203-222. https://doi.org/10.1080/15422119.2019.1673776.
Liu, Z., S. Rochfort, and B. Cocks. 2018. Milk lipidomics: What we know and what we don't. Prog. Lipid Res. 71:70-85. https://doi .org/10.1016/j.plipres.2018.06.002.

Martini, M., I. Altomonte, E. Manica, and F. Salari. 2015. Changes in donkey milk lipids in relation to season and lactation. J. Food Compos. Anal. 41:30-34. https://doi.org/10.1016/j.jfca.2014.12 .019 .

Massouras, T., K. A. Triantaphyllopoulos, and I. Theodossiou. 2017. Chemical composition, protein fraction and fatty acid profile of donkey milk during lactation. Int. Dairy J. 75:83-90.

Mozaffarian, D., and J. H. Wu. 2012. (n-3) Fatty acids and cardiovascular health: Are effects of EPA and DHA shared or complementary? J. Nutr. 142:614S-625S. https://doi.org/10.3945/jn.111 .149633.

Nguyen, M. T. T., J. Kim, N. Seo, A. H. Lee, Y.-K. Kim, J. A. Jung, D. Li, X. H. M. To, K. T. N. Huynh, T. Van Le, B. Israr, A. Nazir, J.-A. Seo, D. Lee, H. J. An, and J. Kim. 2021. Comprehensive analysis of fatty acids in human milk of four Asian countries. J. Dairy Sci. 104:6496-6507. https://doi.org/10.3168/jds.2020-18184.

Pegolo, S. G. Stocco, M. Mele, S. Schiavon, G. Bittante, and A. Cecchinato. 2017. Factors affecting variations in the detailed fatty acid profile of Mediterranean buffalo milk determined by 2-dimensional gas chromatography. J. Dairy Sci. 100:2564-2576. https:// doi.org/10.3168/jds.2016-11696.

Rebechi, S. R., M. A. Vélez, S. Vaira, and M. C. Perotti. 2016. Adulteration of Argentinean milk fats with animal fats: Detection by fatty acids analysis and multivariate regression techniques. Food Chem. 192:1025-1032. https://doi.org/10.1016/j.foodchem.2015 .07.107.

Ruiz-Rodriguez, A., G. Reglero, and E. Ibanez. 2010. Recent trends in the advanced analysis of bioactive fatty acids. J. Pharm. Biomed. Anal. 51:305-326. https://doi.org/10.1016/j.jpba.2009.05.012.

Serafim, V., D. A. Tiugan, N. Andreescu, A. Mihailescu, C. Paul, I. Velea, M. Puiu, and M. D. Niculescu. 2019. Development and validation of a LC-MS/MS-based assay for quantification of free and total omega 3 and 6 fatty acids from human plasma. Molecules 24:360. https://doi.org/10.3390/molecules24020360.

Shokryazdan, P., M. A. Rajion, G. Y. Meng, L. J. Boo, M. Ebrahimi, M. Royan, M. Sahebi, P. Azizi, R. Abiri, and M. F. Jahromi. 2017. Conjugated linoleic acid: A potent fatty acid linked to animal and human health. Crit. Rev. Food Sci. Nutr. 57:2737-2748. https:// doi.org/10.1080/10408398.2015.1060190.

Siurana, A., A. Ferret, M. Rodriguez, B. Vlaeminck, V. Fievez, and S. Calsamiglia. 2018. Strategies to modify the ruminal biohydrogenation of polyunsaturated fatty acids and the production of trans-10, cis-12 C18:2 in vitro. Anim. Feed Sci. Technol. 235:158-165. https: //doi.org/10.1016/j.anifeedsci.2017.11.016.

Sokol, E., T. Ulven, N. J. Færgeman, and C. S. Ejsing. 2015. Comprehensive and quantitative profiling of lipid species in human milk, cow milk and a phospholipid-enriched milk formula by GC and MS/MSALL. Eur. J. Lipid Sci. Technol. 117:751-759. https://doi .org/10.1002/ejlt.201400575.

Teng, F., P. Wang, L. Yang, Y. Ma, and L. Day. 2017. Quantification of fatty acids in human, cow, buffalo, goat, yak, and camel milk using an improved one-step GC-FID method. Food Anal. Methods 10:2881-2891. https://doi.org/10.1007/s12161-017-0852-z.

Thurnhofer, S., and W. Vetter. 2005. A gas chromatography/electron ionization-mass spectrometry-selected ion monitoring method for determining the fatty acid pattern in food after formation of fatty acid methyl esters. J. Agric. Food Chem. 53:8896-8903. https://doi.org/10.1021/jf051468u.

Toral, P. G., G. Hervás, A. Della Badia, R. Gervais, and P. Frutos. 2020. Effect of dietary lipids and other nutrients on milk odd- and branched-chain fatty acid composition in dairy ewes. J. Dairy Sci. 103:11413-11423. https://doi.org/10.3168/jds.2020-18580.

Vlaeminck, B., R. Gervais, M. M. Rahman, F. Gadeyne, M. Gorniak, M. Doreau, and V. Fievez. 2015. Postruminal synthesis modifies the odd- and branched-chain fatty acid profile from the duodenum 
to milk. J. Dairy Sci. 98:4829-4840. https://doi.org/10.3168/jds 2014-9207.

Waktola, H. D., A. X. Zeng, S.-T. Chin, and P. J. Marriott. 2020. Advanced gas chromatography and mass spectrometry technologies for fatty acids and triacylglycerols analysis. Trends Analyt. Chem. 129:115957. https://doi.org/10.1016/j.trac.2020.115957.

Wang, F., M. Chen, R. Luo, G. Huang, X. Wu, N. Zheng, Y. Zhang, and J. Wang. 2021. "Fatty acid profiles of milk from Holstein cows, Jersey cows, buffalos, yaks, humans, goats, camels, and donkeys based on gas chromatography-mass spectrometry", Mendeley Data, V1, https://doi.org/10.17632/j9h7967pcy.1.

Wang, L., X. Li, M. Hussain, L. Liu, Y. Zhang, and H. Zhang. 2020. Effect of lactation stages and dietary intake on the fatty acid composition of human milk (A study in northeast China). Int. Dairy J. 101:104580. https://doi.org/10.1016/j.idairyj.2019.104580.

Wu, T. C., B. H. Lau, P. H. Chen, L. T. Wu, and R. B. Tang. 2010. Fatty acid composition of Taiwanese human milk. J. Chin. Med. Assoc. 73:581-588. https://doi.org/10.1016/S1726-4901(10)70127 -1 .

Yurchenko, S., A. Sats, V. Tatar, T. Kaart, H. Mootse, and I. Jõudu. 2018. Fatty acid profile of milk from Saanen and Swedish Land- race goats. Food Chem. 254:326-332. https://doi.org/10.1016/j .foodchem.2018.02.041

Zhao, P., S. Zhang, L. Liu, X. Pang, Y. Yang, J. Lu, and J. Lv. 2018. Differences in the triacylglycerol and fatty acid compositions of human colostrum and mature milk. J. Agric. Food Chem. 66:45714579. https://doi.org/10.1021/acs.jafc.8b00868.

Zou, X., J. Huang, Q. Jin, Z. Guo, Y. Liu, L. Cheong, X. Xu, and X. Wang. 2013. Lipid composition analysis of milk fats from different mammalian species: Potential for use as human milk fat substitutes. J. Agric. Food Chem. 61:7070-7080. https://doi.org/ 10.1021/jf401452y.

\section{ORCIDS}

Fengen Wang ๑ https://orcid.org/0000-0002-7236-4669

Guoxin Huang (ํ) https://orcid.org/0000-0003-3898-8002

Nan Zheng (๑) https://orcid.org/0000-0002-5365-9680

Yangdong Zhang $\odot$ https://orcid.org/0000-0002-5256-0531

Jiaqi Wang @ $\odot$ https://orcid.org/0000-0001-8841-0124 